\title{
A simple technique for correlating film and EMG in locomotion studies
}

\author{
ANDREW K. CHAN, STANLEY RASMUSSEN, and G. E. GOSLOW, JR. \\ Departments of Engineering, Physical Education, and Biological Sciences, \\ Northern Arizona University, Flagstaff, Arizona 86001
}

\begin{abstract}
Two recurring problems hampering investigations of muscular activity patterns during locomotion are electrical "noise" and movement artifact in the electromyographic (EMG) signal and proper synchronization of behavioral events to the EMG. A relatively simple technique for achieving these ends is presented. Vigorous locomotor movements of unrestrained animals are recorded on 16-mm film and synchronized with one or more EMGs from selected muscles with little electrical noise or movement artifact interference. The system employs telemetry which is compared to hardwire techniques.
\end{abstract}

Techniques for correlating electromyogram (EMG) with animal behavioral events are of interest to vertebrate morphologists and others (cf. Cohen \& Gans, 1975; English, 1975; Herring, 1973; Kallen \& Gans, 1972; Liem \& Osse, 1975).

Of course, the EMG methods vary. With a hardwired preparation, EMG recording electrodes are connected to the preamplifier through long cables to allow freedom of movement. This method is relatively inexpensive, and it allows several muscles to be monitored simultaneously. On the other hand, this technique has several drawbacks: (1) The long electrode leads can act as antennas for low-frequency electrical noise; (2) the movements of large animals may be restricted by the length of the leads, especially when a Faraday cage is used to shield off unwanted electrical noise; (3) the movements of the leads can create extensive movement artifacts while the animal is in motion. These artifacts appear in the EMG recordings as overriding ac signals with frequencies equal to the repetition rate of the motion of the animal.

To avoid restrictions of the animals' behavior during recording, various methods of signal telemetry may be employed. In our studies of the EMG activity of select hind-limb muscles of the striped skunk and domestic cat during unrestrained locomotion, muscle activity patterns were successfully telemetered and synchronized with other behaviors recorded on $16-\mathrm{mm}$ film. Though various published accounts are available for EMG telemetry and film synchronization (Bevan, 1972; Brandell, 1970), the systems are often quite expensive, or they are described in such technical terms as to be difficult to duplicate for the researcher without expertise in elec-

The first author's present address is Department of Electrical Engineering, Texas A \& M University, College Station, Texas 77843. Much of the early development of this system was supported by the Organized Research Committee, Northern Arizona University. tronics. A relatively simple recording system is described here.

Figure 1 illustrates our arrangement. The camera is the Bolex H16. Synchronization of the EMG with movements recorded on film (to 64 frames/sec) is accomplished by a magnetic reed switch attached to the drive shaft of the camera as described by Gans (1966). Each time the shutter of the camera is opened, a small ( $1 \mathrm{mV}$ or less) inductive pulse is generated from the switch (Industrial Camera Company, Old Hill Road, Epsom, New Hampshire 03239: \$50). This pulse is produced by a small coil positioned near the circular path of a revolving magnet attached to the drive shaft of the camera. The pulse signal can be put directly into a recorder with a high-gain preamplifier; the recording output serves as a timing marker. Because of the twinlead arrangement of the switch, $60-\mathrm{Hz}$ noise picked up by the leads is usually high and the pulse signal from the camera is buried under the noise. To overcome this problem, a stroboslave (General Radio, Type 1539-A, $\$ 215$ ) is connected in parallel with the recorder such that the discharge of the capacitor in strobe boosts the signal to a higher level. A properly grounded metallic mesh lead sleeve is used to shield the $60-\mathrm{Hz}$ noise. With this arrangement, only a medium-gain preamplifier is needed to record the pulse signal. (An alternative to this timing arrangement is to use an opto-electronic system

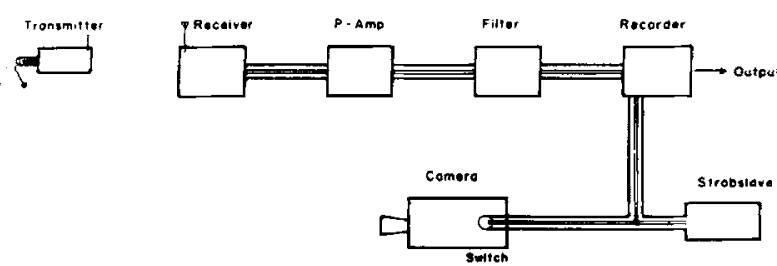

Figure 1. Block diagram of the movie camera and EMG telemetry system. (Cost: transmitter, \$200; receiver, \$650; preamplifier, $\$ 360$; filter, $\$ 1,000$; recorder, $\$ 2,700$; stroboslave, \$200.) 
with a 741 op amp to boost the signal. Total cost can be reduced to $\$ 60$.) Our method requires the EMG channel to be turned on prior to the first frame of the motion picture sequence. Both the EMG signal and the pulse signal from the camera must be recorded on the same chart in order to properly correlate behavior events with EMG.

Two problems normally associated with recording EMGs from large muscles via implanted electrodes are movement artifact and contamination from unwanted electrical noise. Movement artifact may be reduced significantly by introducing a bandpass filter in the EMG signal path. The bandpass filter (General Radio, Type 1952, $\$ 1,000$ ) can be adjusted so that the frequency spectrum of the EMG is retained in the output. The bandpass filter has better than $30 \mathrm{~dB} /$ octave roll off at both high and low corner frequencies. In our studies the corner frequencies were $20-200 \mathrm{~Hz}$. These choices were based on observations of raw signals from a cat muscle and the studies of Hayes (1960). The results are shown in Figure 2. The bandpass filter, coupled with the monopolar electrode technique described by Engberg (1964), effectively reduce the movement artifact for such vigorous behavior as galloping and jumping. Briefly, the monopolar technique requires the use of a rectal ball (@1 cm diam) as the indifferent and a fine enamel insulated copper or silver wire $(.1 \mathrm{~mm}$ OD) (California Fine Wire Company, Gardena) inserted into the muscle with a 25-g hypodermic needle, or modified needle after Osse, Oldenhave, and Van Shie (1972). The recording electrode and rectal ball are connected to a small-diameter coaxial cable and plugged into the transmitter carried in an elastic band about the animal's thorax. Anaesthesia for ball and electrode placement is unnecessary; following a few training sessions with the elastic band in position, the skunks and cats appear to

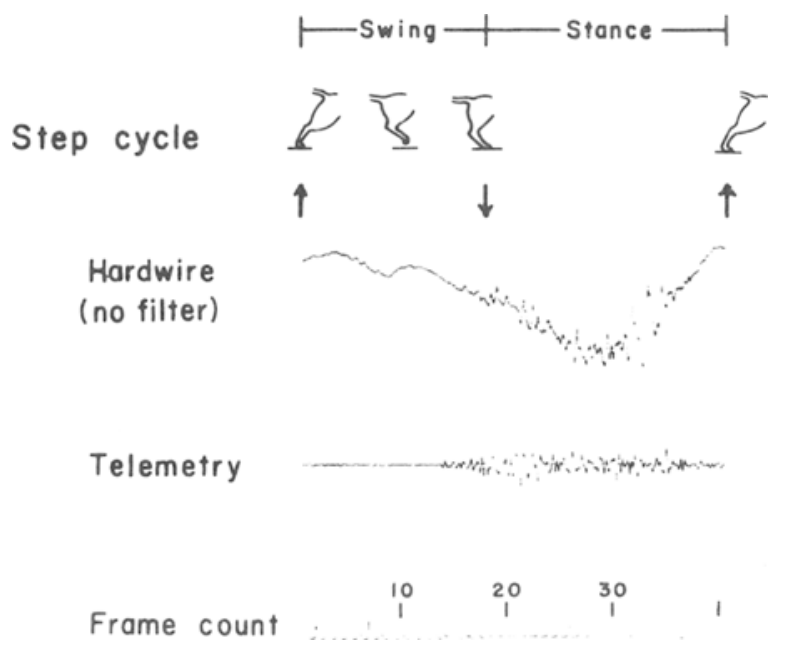

Figure 2. EMG signals of a typical cat ankle extensor recorded by EMG hardwiring and telemetry techniques. Above: typical step cycle. Below: camera signals with EMG recorded simultaneously for precise correlation of muscle activity locomotor events. move normally. There are no problems with inserting the rectal ball, and the cat need not be declawed.

Suppression of unwanted electrical noise, such as $60 \mathrm{~Hz}$, is accomplished by grounding all instruments at one point and by utilizing coaxial or shielded cable to route the signal from the electrode site to the transmitter. At the output of the receiver, a three-conductor system (see Figure 1) transfers the signal to the recorder. Unwanted noise is suppressed to less than $1 \mathrm{mV}$, and the signal-to-noise ratio is often better than 10 . A $60-\mathrm{Hz}$ notch filter may be used in the preamplifier to overcome $60-\mathrm{Hz}$ noise if the frequency of the EMG signals is of no interest.

Multichannel capability of this system has also been tested. Complication in multichannel transmission and recording, such as cross-talk and frequency interference, are minimal.

The telemetry equipment is available from NarcoBio Systems, Houston, Texas. The frequency adjustable transmitter (FM-1100E2, \$200) weighs $18 \mathrm{~g}$ with batteries. The receiver (FM-1 100-6, \$650) is connected to a preamplifier (Grass Instruments Company, P511, $\$ 360$ ), which in turn is connected to the bandpass filter. The filter output goes to one channel of the chart recorder (Hewlett Packard, 7402A, \$2,400; mediumgain preamplifier, $17401 \mathrm{~A}, \$ 300)$. Thus, total cost for first EMG channel (including the reed switch and stroboslave) is about $\$ 5,175$.

\section{REFERENCES}

Bevan, R. J. A simple camera synchronizer for combined cinephotography and electromyography kinesiology for use with a pen recorder. Research Quarterly, 1972, 43, 105-112.

BRANDELL, B. R. An electromyographic-cinematrographic study of muscles of index finger. Archive Physical Medicine and Rehabilitation, 1970, 5, 278-285.

Cohen, A. H., \& Gans, C. Muscle activity in rat locomotion: Movement analysis and electromyography of the flexors and extensors of the elbow. Journal of Morphology, 1975 , 146, 177-196.

Engrerg, I. Reflexes to foot muscles in the cat. Acta Physiologica Scandinavia, 1964, 62, Supplement 235.

ENGLish, A. Electromyography of shoulder muscles during unrestrained locomotion in the cat. American Zoology, $1975,15,800$.

Gavs, C. An inexpensive arrangement of movie camera and electronic flash as a tool in the study of animal behavior. Animal Behaviour, 1966, 14, 11-12.

HAYES, K. J. Wave analysis of tissue noise and muscle action potentials. Journal of Applied Physiology, 1960, 15, 749-752.

HERring, S. Physiology of feeding minature pigs. Journal of Morphology, 1973, 141, 427-460.

Kallen, F. C., \& Gans, C. Mastication in the little brown bat, Myotis lucifgus. Joumal of Morphology, 1972, 136, 385-420.

LIEM, K. F., \& OSSE, J. W. M. Biological versatility, evolution, and food resource exploitation in African cichlid fishes. American Zoology, 1975, 15, 427-454.

Osse, J. W. M., Oldenhave, M., \& Van Shie, B. A new method of insertion of wire electrodes in electromyography. Electromyography, 1972, 12, 59-62.

(Received for publication February 1, 1977; revision accepted A pril 12, 1977.) 